\title{
Two Classifiers Based on Serum Peptide Pattern for Prediction of HBV-Induced Liver Cirrhosis Using MALDI-TOF MS
}

\author{
Yuan Cao, ${ }_{1}^{1}$ Kun He, ${ }^{2}$ Ming Cheng, ${ }^{3}$ Hai-Yan Si, ${ }^{1}$ He-Lin Zhang, ${ }^{1}$ Wei Song, ${ }^{2}$ Ai-Ling Li, ${ }^{2}$ \\ Cheng-Jin $\mathrm{Hu},{ }^{1}$ and $\mathrm{Na} \mathrm{Wang}^{2}$ \\ ${ }^{1}$ Department of Laboratory Medicine, Jinan Military General Hospital, Jinan, Shandong 250031, China \\ ${ }^{2}$ Institute of Basic Medical Sciences, National Center of Biomedical Analysis, Beijing 100850, China \\ ${ }^{3}$ Department of Neurology, Jinan Military General Hospital, Jinan, Shandong 250031, China
}

Correspondence should be addressed to Cheng-Jin Hu; hcj6269@163.com and Na Wang; nawna@126.com

Received 5 October 2012; Accepted 9 January 2013

Academic Editor: Anne Hamburger

Copyright (C) 2013 Yuan Cao et al. This is an open access article distributed under the Creative Commons Attribution License, which permits unrestricted use, distribution, and reproduction in any medium, provided the original work is properly cited.

\begin{abstract}
Chronic infection with hepatitis B virus (HBV) is associated with the majority of cases of liver cirrhosis (LC) in China. Although liver biopsy is the reference method for evaluation of cirrhosis, it is an invasive procedure with inherent risk. The aim of this study is to discover novel noninvasive specific serum biomarkers for the diagnosis of HBV-induced LC. We performed bead fractionation/MALDI-TOF MS analysis on sera from patients with LC. Thirteen feature peaks which had optimal discriminatory performance were obtained by using support-vector-machine-(SVM-) based strategy. Based on the previous results, five supervised machine learning methods were employed to construct classifiers that discriminated proteomic spectra of patients with HBVinduced LC from those of controls. Here, we describe two novel methods for prediction of HBV-induced LC, termed LC-NB and LC-MLP, respectively. We obtained a sensitivity of $90.9 \%$, a specificity of $94.9 \%$, and overall accuracy of $93.8 \%$ on an independent test set. Comparisons with the existing methods showed that LC-NB and LC-MLP held better accuracy. Our study suggests that potential serum biomarkers can be determined for discriminating LC and non-LC cohorts by using matrix-assisted laser desorption/ionization time-of-flight mass spectrometry. These two classifiers could be used for clinical practice in HBV-induced LC assessment.
\end{abstract}

\section{Introduction}

Patients with liver cirrhosis (LC) caused by chronic hepatitis $B$ virus (HBV) are at high risks of developing hepatocellular carcinoma (HCC) [1-4]. In China, the proportion of people infected with $\mathrm{HBV}$ is higher than that in other countries with an estimated 120 million $[5,6]$. During a 5 -year period, $10 \%-$ $20 \%$ of patients with chronic hepatitis developed cirrhosis, and $6 \%-15 \%$ of the people with cirrhosis and chronic hepatitis progressed to HCC, among whom 5-year survival is less than 5\% [7]. At present, liver biopsy has been the "gold standard" for evaluation of stage of liver fibrosis and cirrhosis [8], but it is limited as it is an invasive procedure with significant expense, manpower issues, and some risks. Furthermore, intra- and interobserver variations for interpretation of biopsies are $10 \%-20 \%$, even among experienced pathologists [9]. For this reason, developing diagnosis biomarkers of LC is an alternative way for assessing prognosis and candidacy for treatment in patients with chronic liver disease. Over the past decade, attempts have been made to develop noninvasive methods to assess LC, including physical approaches and biological approaches. Physical approaches include 2$\mathrm{D}$ acoustic radiation force impulse imaging (ARFI), 3-D magnetic resonance (MR) elastography, and 1-dimensional ultrasound transient elastography (TE) [10]. ARFI can be easily implemented, but it has a limited range compared with TE [11]. TE analysis has excellent inter- and intraassay agreements, but its applicability (80\%) is not as good as that of serum biomarkers [12]. Although MR elastography can analyze almost the entire liver, it is too expensive and time consuming to use in routine practice [8]. In recent years, serum-based tests of liver cirrhosis have attracted more attention, such as the aspartate to platelet ratio index [13] and the FibroTest [8, 14-18]. However, most of these studies on 
biomarkers of liver cirrhosis have been conducted in chronic hepatitis $\mathrm{C}$, and few data are available on the applicability of this approach to patients infected with HBV [19]. Meanwhile, some serum biomarkers related to the fibrogenic process, such as hyaluronic acid, may be confounded by associated diseases with fibrosis in other organs [20].

Recently, proteomics studies using high-throughput spectrometric methods such as matrix-assisted laser desorption/ionization time-of-flight mass spectrometry (MALDITOF MS) and surface-enhanced laser desorption/ionization time-of-flight mass spectrometry (SELDI-TOFMS) have proved possible methods for the identification of new disease biomarkers [21]. Up to now, advances based on proteomics have been made in the understanding of hepatitis and liver cirrhosis. Zhu et al. [22] proposed two serum biomarkers for HBV-induced LC using SELDI technology. They obtained a sensitivity of $80.0 \%$ for all LC patients and a specificity of $81.8 \%$ for all noncirrhotic cohorts. Bozdayi et al. [15] provided similar results with a sensitivity of $83.3 \%$ and a specificity of $85.1 \%$. However, establishment of serum peptide pattern for predicting HBV-induced LC from noncirrhotic cohorts remains challenging. Consequently, the objective of this work was to identify serum peptidome signatures associated with liver cirrhosis by using the MALDI-TOF MS and to construct classifiers for predicting liver cirrhosis in patients with HBV infection.

\section{Materials and Methods}

2.1. Patients and Sample Collection. From December 2009 to August 2010, a total of 162 serum samples including 44 LC patients with chronic hepatitis B (CHB), 46 patients with $\mathrm{CHB}$, and 72 healthy individuals were collected with informed consent in Jinan Military General Hospital (Jinan, China). The design of the study was approved by the Hospital Ethical Committee. Two groups of consecutive subjects were enrolled. The first group consisted of patients with HBVinfected LC. LC patients were diagnosed mainly depending on clinical history, physical examination, laboratory results, and ultrasonographic and/or computed tomographic imaging with liver biopsy [23]. The second group consisted of patients with $\mathrm{HBV}$ and healthy individuals. Patients with HBV were diagnosed based on HBsAg (+), HBeAg (+), and HBVDNA (+), as well as abnormal liver biochemistry. Healthy individuals showed no risk factors for viral hepatitis, no history of liver disease, and normal liver function.

Blood sampling from patients was done before the initiation of specific therapy. The blood samples were collected in $5 \mathrm{~mL}$ BD Vacutainers without anticoagulation and allowed to clot at room temperature for up to $1 \mathrm{hr}$ [24]. The sera were obtained by centrifugation at $2000 \mathrm{rpm}$ for $15 \mathrm{~min}$ and were immediately frozen and stored at $-80^{\circ} \mathrm{C}$ until testing. It took no more than $60 \mathrm{~min}$ from serum collection to frozen storage.

\subsection{Peptide Separation and MALDI-TOF MS Analysis.} Copper-chelated magnetic beads and solutions were used for extracting peptides from serum samples [25]. In brief, $5 \mu \mathrm{L}$ serum was mixed with $5 \mu \mathrm{L}$ beads and $20 \mu \mathrm{L}$ of binding solution. The beads were washed three times with $100 \mu \mathrm{L}$ of wash solution after $10 \mathrm{~min}$ incubation [26]. Then, $20 \mu \mathrm{L}$ of eluent buffer were used to elute the bound peptides. After mixing with $1 \mu \mathrm{L}$ of CHCA matrix solution, the eluent was spotted onto a $600 \mu \mathrm{m}$-diameter spot size 384 MTP target plate (Bruker Daltonik, Germany) until dry. The peptide calibration standard was applied to target spots for external calibration of the instrument in the same matrix. To obtain the peptidome of serum samples, the analysis of the processed samples was performed by an Autoflex MALDI-TOF MS (Bruker Daltonics, Germany) equipped with a pulsed ion extraction ion source.

Serum pools were analyzed from healthy volunteers 5 times for reproducibility. Within-run assays were carried out by using MALDI-TOF MS. The mean value and coefficient of variance $(\mathrm{CV})$ were calculated, respectively.

2.3. Data Preprocessing and Feature Selection. Three bioinformatics tools were employed for data processing and analysis, including ClinProt Tools software 2.2 (Bruker Daltonik, Germany), flexAnalysis (Bruker Daltonics, Germany), and Waikato Environment for Knowledge Analysis (WEKA) [27]. First, mass spectra of all serum sample data derived from LC and control were preprocessed via the ClinProt, including baseline subtraction of spectra, normalization and recalibration of a set of spectra, and internal peak alignment by using prominent peaks. Then, the processed data were stored in BioSunMS [28] and were prepared for feature selection in WEKA. In BioSunMS, independent training set $(n=81)$ and the test sets $(n=81)$ were created. Based on the training set, peak statistics were done by the SVM-based strategy in WEKA. In feature selection, "cross-validation" was selected as the attribute selection mode, and tenfold splits as default were performed. Finally, we obtained a list of peaks sorted along the statistical difference between two classes (e.g., LC and control), which was used for further data analysis.

2.4. Classifier Construction and Evaluation. To predict liver cirrhosis, five classifiers were constructed based on the training set using five supervised machine learning methods (naïve Bayes: NB: multilayered perceptron: MLP: support vector machine: SVM; C4.5 decision tree; DT; classification and regression tree; CART) in WEKA, respectively. A 10fold cross-validation was performed to avoid model-specific overfitting. To perform cross-validation, all the records were randomly divided into ten parts; nine sets were used for training and the rest one for testing. The process was repeated ten times and the accuracy for true, false and total accuracy was calculated. The final accuracy is the average of the accuracy in all ten tests. With the test set (22 from patients with LC, 23 from patients infected with $\mathrm{HBV}$, and 36 from healthy individuals), we evaluated the generalization performance of the five classifiers by considering the number of correctly classified (true positives, TPs, and true negatives, TNs) and incorrectly classified (false positives, FPs, and false negatives, FNs) samples in the test set. Accuracy (ACC), sensitivity (SE), and specificity (SP) were also calculated. To evaluate the performance of a range of classifiers, StAR [29] was 
Table 1: Participant demographics.

\begin{tabular}{lcccccccccc}
\hline & \multicolumn{3}{c}{ CHB cirrhosis } & \multicolumn{3}{c}{ Control } \\
& \multicolumn{3}{c}{ HBV with LC } & \multicolumn{3}{c}{ HBV without LC } & \multicolumn{3}{c}{ Healthy individuals } & \multicolumn{3}{c}{ Total } \\
& Training & Test & Total & Training & Test & Total & Training & Test & Total & \\
\hline Total number of patients & 22 & 22 & 44 & 23 & 23 & 46 & 36 & 36 & 72 & 162 \\
Mean (range) age in years & 49.28 & 51.75 & 50.16 & 47.83 & 46.39 & 47.11 & 47.08 & 47.97 & 47.55 & 48.12 \\
& $(25-68)$ & $(32-71)$ & $(25-71)$ & $(28-59)$ & $(28-61)$ & $(28-64)$ & $(35-55)$ & $(32-56)$ & $(32-56)$ & $(25-71)$ \\
Sex (male : female) & $16: 6$ & $17: 5$ & $33: 11$ & $15: 8$ & $17: 6$ & $32: 14$ & $19: 17$ & $18: 18$ & $37: 35$ & $102: 60$ \\
\hline
\end{tabular}

TABLE 2: Top twenty peptide patterns selected between each among the three groups.

\begin{tabular}{lcc}
\hline HBV with LC versus & $\begin{array}{c}\text { HBV with LC versus } \\
\text { healthy individuals }\end{array}$ & $\begin{array}{c}\text { HBV without LC } \\
\text { versus healthy } \\
\text { individuals }\end{array}$ \\
\hline 3880 & 4165 & 2670 \\
$6945^{*}$ & $6945^{*}$ & 4165 \\
$4202^{*}$ & 4133 & 4061 \\
4267 & 2670 & 4298 \\
2929 & 4465 & 6451 \\
$807^{*}$ & $1928^{*}$ & $1449^{*}$ \\
3889 & $916^{*}$ & $4207^{*}$ \\
3027 & $1536^{*}$ & 3260 \\
8946 & $4207^{*}$ & 2929 \\
6451 & $4281^{*}$ & 6636 \\
$1017^{*}$ & $1011^{*}$ & 8946 \\
1531 & $1449^{*}$ & 876 \\
2080 & 853 & $2551^{*}$ \\
1942 & 4789 & 5906 \\
6974 & 1933 & 3339 \\
6649 & $2551^{*}$ & $3951^{*}$ \\
1933 & 2080 & 2035 \\
$4281^{*}$ & 1531 & 6649 \\
1043 & 860 & 4119 \\
$1785^{*}$ & $807^{*}$ & 4353 \\
\hline 625 &
\end{tabular}

${ }^{*}$ Represents peptide in the selected peptide pattern.

used to plot receiver operator curves (ROCs) and statistical comparison of area under curves.

\section{Results}

3.1. Reproducibility of the Autoflex System. The reproducibility with ten mixed serum samples from the healthy controls was evaluated by the Autoflex MALDI-TOF MS. The mixed serum samples were spotted on five spot WCX magnetic beads. Overall, the Autoflex revealed the mean CV (19.8\%) for within-run assay (Figure 1).

3.2. Dataset and Feature Selection. To screen serum peptides for prediction of liver cirrhosis in chronic hepatitis B infection, we used MALDI-TOF MS to analyze serum samples of
$44 \mathrm{LC}$ patients and 118 non-LC individuals (46 HBV infected and 72 healthy). The complete mass spectrum comparison of serum samples between LC and non-LC groups is listed in Figure 2. A total of 235 peaks with $\mathrm{m} / z$ between 800 and $10000 \mathrm{Da}$ were obtained from the 162 serum samples. Fifty percent of subjects serum spectra were used as training set $(n=81)$ and others as test set $(n=81)$ (Table 1$)$. After data preprocessing, feature variables were evaluated by using the SVM-based strategy and ranked by the square of the weight assigned by the SVM. A panel of 14 peptides was selected for classifier construction based on the training set, which have $\mathrm{m} / \mathrm{z}$ ratios of $807,916,1011,1017,1449,1536,1785,1928,2551$, $3951,4202,4207,4281$, and $6945 \mathrm{Da}$. Because the samples were from HBV-infected patients with and without LC and healthy individuals, we thus compared the peptide patterns between each among the three groups. With the SVM strategy, the top twenty peptides marked differently between two groups were listed in Table 2. We found that the peptide of $3951 \mathrm{Da}$ just revealed significant difference between HBV without LC and healthy individuals. Therefore, we excluded this peptide from the peptide pattern.

3.3. Construction and Evaluation of the Five Classifiers. To predict LC, five classifiers including LC-NB, LC-MLP, LCSVM, LC-DT, and LC-CART were constructed by five supervised machine learning methods, that is, NB, MLP, SVM, DT, and CART, respectively. To evaluate the performance of the five classifiers, we applied a preliminary test by tenfold crossvalidation on the training set (Table 3). LC-MLP brought the best results with ACC of $97.5 \%$, SE of $95.5 \%$, and SP of $98.3 \%$. The ACC, SE, and SP of LC-NB were $93.8 \%, 86.4 \%$, and $96.6 \%$ respectively. LC-SVM indicated the comparable results with ACC of $90.1 \%$ and SP of $96.6 \%$ but gave the poorer SE of $72.7 \%$. LC-DT and LC-CART displayed similar results on accuracy and specificity (ACC, 86.4\%, SE, 68.2\%, and SP, 93.2\%, for LC-DT, and ACC, 85.2\%, SE, 59.1\%, and SP, 94.9\%, for LC-CART).

The ability of a classifier to discriminate data correctly in the test set is known as its generalization performance [30]. We thus compared the generalization performance of a series of classifiers by plotting their performance on the test set in ROC space (Figure 3). The LC-MLP classifier (Table 4 and Figure 3 ) showed the best results on the test set, with SE of $90.9 \%$ and SP of $94.9 \%$ (overall accuracy $93.8 \%$ ), while LC-NB presented the highest SP of $96.6 \%$ (overall accuracy $93.8 \%$ ). The AUCs are $0.977,0.973,0.853,0.825$, and 

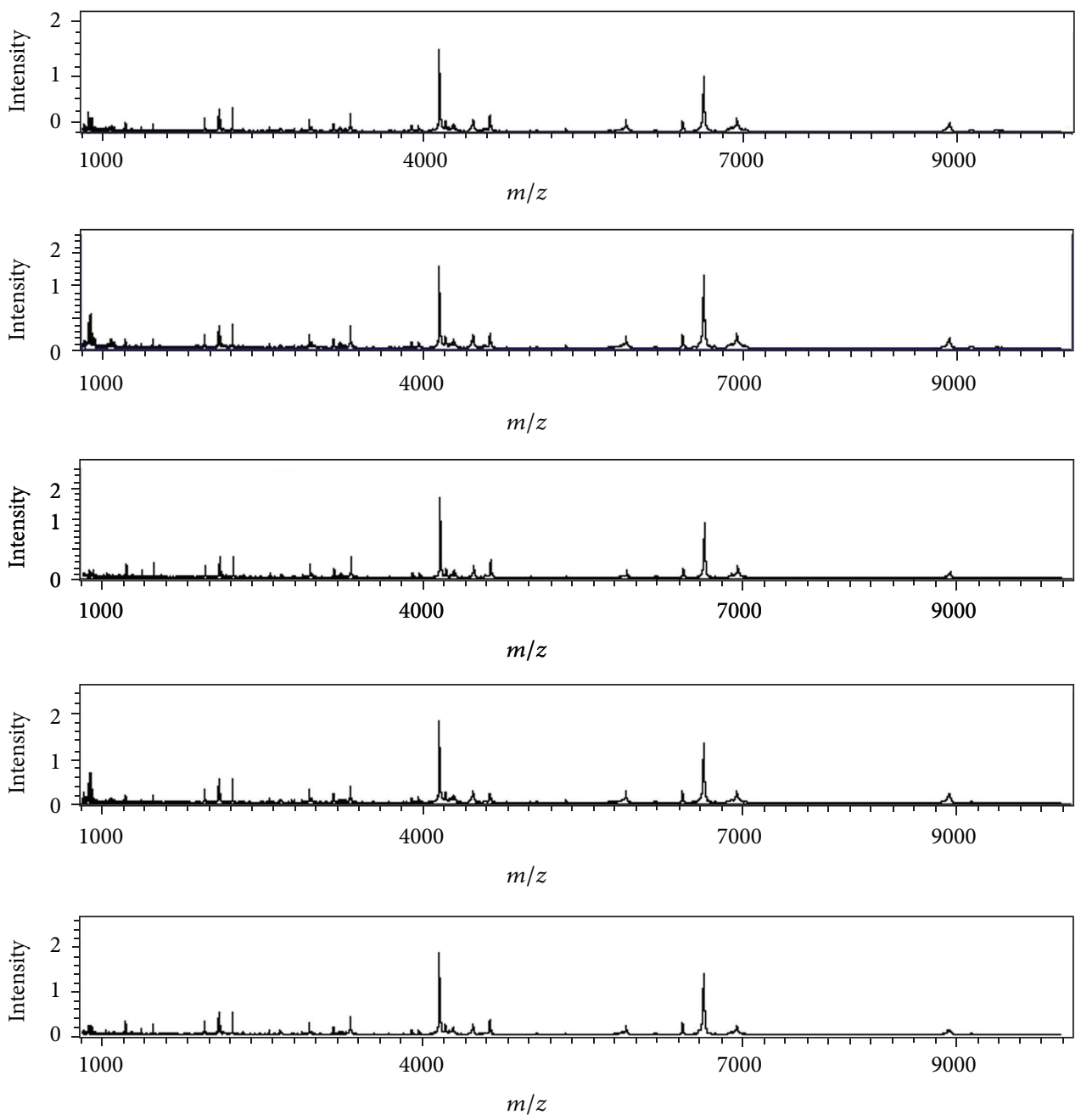

FIGURE 1: Spectra illustrating reproducibility of 5 separate analyses from controls.

0.733 for LC-NB, LC-MLP, LC-SVM, LC-DT, and LC-CART, respectively. The difference of AUCs between LC-NB and LCMLP has no statistical significance at a default significant level $(P=0.7871)$.

\section{Discussion}

In this study, we obtained thirteen serum peptides for the prediction of $\mathrm{HBV}$-induced liver cirrhosis by statistical comparison of serum peptide pattern of LC and control with MALDI-TOF MS. Subsequently, we investigated five supervised machine learning methods for predicting liver cirrhosis. We found that LC-NB and LC-MLP gave better results (AUC > 0.950) among the five classifiers. Our results indicate that the two classifiers are useful tools for predicting $\mathrm{HBV}$-infected liver cirrhosis through analysis of the serum peptide pattern.

LC is defined as the histological development of regenerative nodules surrounded by fibrous bands in response to chronic liver injury, which is mainly caused by $\mathrm{HBV}$ in China $[10,31]$. The latest European Association for the
TABLE 3: Comparison of performance via tenfold cross-validation.

\begin{tabular}{lccccccc}
\hline Classifiers & TP & TN & FP & FN & ACC (\%) & SE (\%) & SP (\%) \\
\hline LC-NB & 19 & 57 & 2 & 3 & 93.8 & 86.4 & 96.6 \\
LC-MLP & 21 & 58 & 1 & 1 & 97.5 & 95.5 & 98.3 \\
LC-SVM & 16 & 57 & 2 & 6 & 90.1 & 72.7 & 96.6 \\
LC-DT & 15 & 55 & 4 & 7 & 86.4 & 68.2 & 92.7 \\
LC-CART & 13 & 56 & 3 & 9 & 85.2 & 59.1 & 94.9 \\
\hline
\end{tabular}

TABLE 4: Performance of the five classifiers on the test set.

\begin{tabular}{lcccccccc}
\hline Classifiers & TP & TN & FP & FN & ACC (\%) & SE (\%) & SP (\%) & AUC \\
\hline LC-NB & 19 & 57 & 2 & 3 & 93.8 & 86.4 & 96.6 & 0.977 \\
LC-MLP & 20 & 56 & 3 & 2 & 93.8 & 90.9 & 94.9 & 0.973 \\
LC-SVM & 17 & 55 & 4 & 5 & 88.9 & 77.3 & 92.7 & 0.853 \\
LC-DT & 17 & 53 & 6 & 5 & 86.4 & 77.3 & 89.8 & 0.825 \\
LC-CART & 14 & 48 & 11 & 8 & 76.5 & 63.6 & 81.4 & 0.733 \\
\hline
\end{tabular}

Study of the Liver (EASL) treatment guidelines on HBV in 2009 recommend that abnormal ALT levels together with 


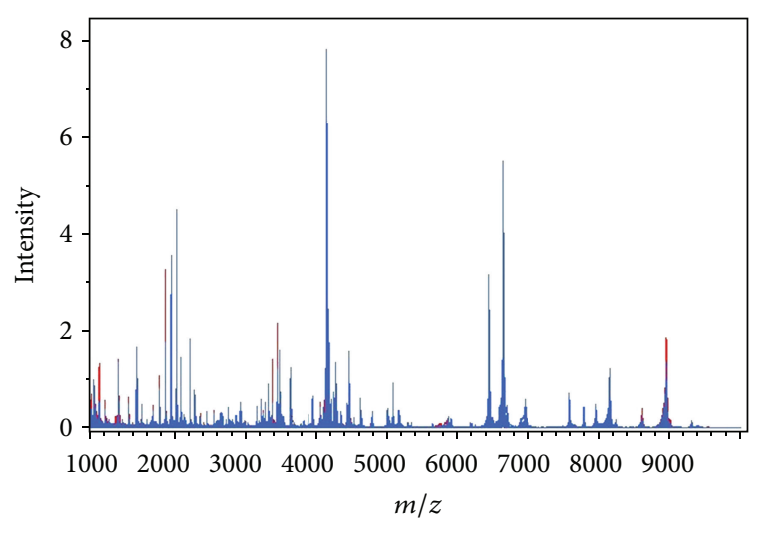

FIGURE 2: Complete mass spectrum of serum samples between HBV-cirrhosis and non-LC groups in the $800-10,000 \mathrm{~m} / z$ range. Red line represents HBV-cirrhosis group; Blue line represents non-LC group.

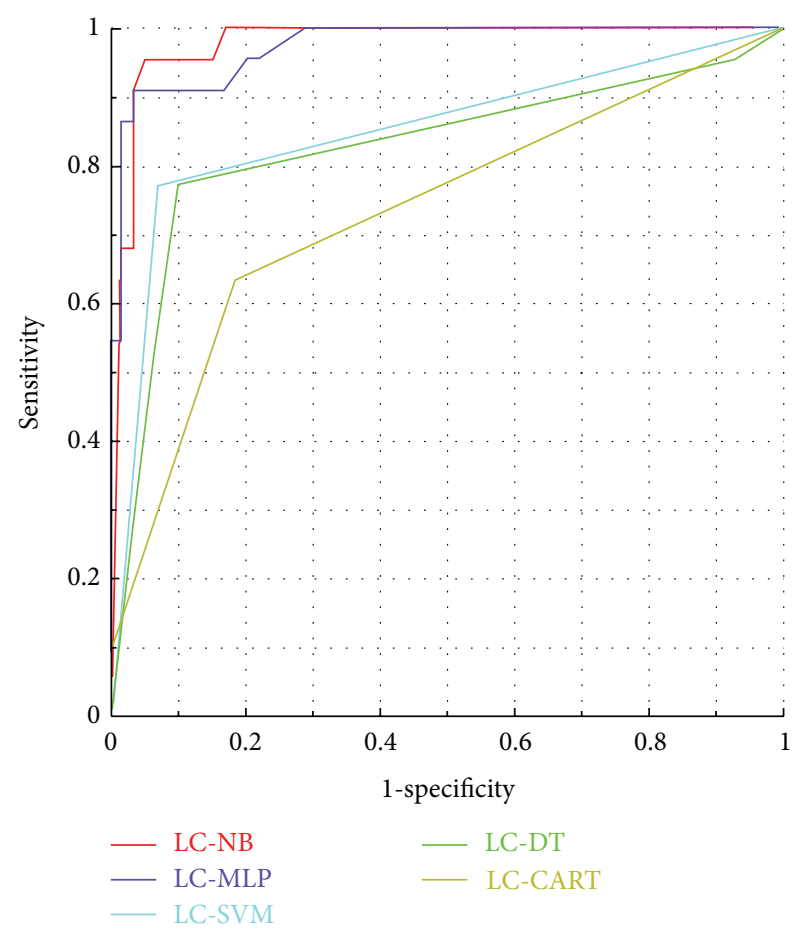

FIgure 3: Classifier performances in ROC space. Red line shows ROC curve of LC-NB. Blue line represents ROC curve of LC-MLP. Cyan line represents LC-SVM. Green line represents LC-DT. Yellow line represents LC-CART. The areas under curve are 0.977, 0.973, 0.853, 0.825, and 0.733 for LC-NB, LC-MLP, LC-SVM, LC-DT, and LC-CART, respectively.

HBV DNA levels of more than 2,000 IU/mL should be evaluated further by liver biopsy [32]. Although histological assessment provides valuable information on the degree of necroinflammation and fibrosis in such patients, it is an invasive procedure associated with a finite albeit small risk of severe complications of $0.5 \%$, patient discomfort, and expense [9]. Therefore, there is a need for a simple, reliable, and noninvasive alternative method for regular monitoring of disease progression. MALDI-TOF MS analysis is a new potential tool for human diseases diagnosis. The low molecular weight proteome or peptidome found in biological fluids can be used to discriminate between patients with or without disease [33]. In addition, combinations of candidate proteins/peptides can often create a more robust test when individual proteins/peptides fail to discriminate between two groups [34]. In this study, we developed a novel approach to predict LC patients infected with HBV based on bead extraction and MALDI-TOF MS analysis. This is a simple, available, and noninvasive procedure compared with other diagnostic approaches. Additionally, the two classifiers, LC-NB and LC-MLP, could differentiate patients with liver cirrhosis from patients with $\mathrm{HBV}$ infection or healthy individuals with high sensitivity (86.4\%-90.9\%) and specificity (94.9\%-96.6\%). Such accuracy compares favorably with models derived from SELDI or MALDI analysis in similar study. In Zhu's report [22], the researchers were able to predict $\mathrm{HBV}$-induced liver cirrhosis with $80 \%$ sensitivity and $82 \%$ specificity using SELDI protein chips. Poon and his colleagues [9] built artificial neural network (ANN) fibrosis index for predicting the degree of fibrosis using SELDI ProteinChip arrays. Their accuracy for prediction of cirrhosis reached $89 \%$. Cui et al. [23] obtained a sensitivity of $91.7 \%$ and specificity of $94.3 \%$ with a SELDI-based serum decision tree classification model from 18 patients with $\mathrm{HBV}$-induced liver cirrhosis and 52 healthy controls. Although their results were better, the HBV-infected patients were not included in the control group.

Feature selection is an important step of data preprocessing [30]. In this work, we filtered fourteen significantly different peptides between HBV with LC and control (HBV without LC and healthy individuals). Then, we compared the peptide patterns between each among the three groups using the SVM strategy (Table 2). Six peptides from the peptide pattern including 6945, 4202, 807, 1017, 4281, and $1785 \mathrm{Da}$ showed obvious difference between HBV with LC and HBV without LC. Nine of the fourteen peptides selected including $6945,1928,916,807,1536,4207,4281,1011,1449$, and $2551 \mathrm{Da}$ showed significant difference between HBV with LC and healthy individuals. Among the fourteen peptides selected, the peptides of 6945,4281 , and $807 \mathrm{Da}$ in HBV with LC show higher level than that of HBV without LC and healthy individuals. They may be potential serum biomarkers during LC development in CHB patients. During our analysis, the gender was excluded from the feature variables for the reason that the male : female ratio is not significantly different between HBV with LC and HBV without LC ( Table 1). After gender was added to the prediction model as one feature, we did not obtain better results. Meanwhile, the gender feature got lower score for feature selection using the SVM strategy.

Classifier performance depends greatly on the characteristics of the data to be classified. There is no single classifier that works best on all given problems. Therefore, in this study, five supervised machine learning methods were employed to construct classifiers for predicting LC. First, we compared their classification accuracy on the training set by 10 -fold cross-validation. The ACCs were 93.8\%, 97.5\%, 90.1\%, 86.4\%, 
and $85.2 \%$ for LC-NB, LC-MLP, LC-SVM, LC-DT, and LCCART, respectively. Because a subset of features that have high classification accuracy on the training set may not have good generalization properties [35], we thus applied our trained classifiers to the test set to avoid biases. The fact that the small decrease in accuracy was obtained on the test set demonstrates the generalization performance of the classifiers. Among the five classifiers, the AUCs of LC-NB and LC-MLP were better. Meanwhile, the difference of AUCs between LC-NB and LC-MLP has no statistical significance $(P=0.7871)$. As a result, both of them were selected as our prediction classifiers in this study (Table 4 and Figure 3).

When we compared the results of the aforementioned studies with that of this study, we found that no protein/peptide peak can be identified with same molecular mass. Possible reasons for this contradiction may lie in the following aspects. First, prefractionation strategies and detection technology were used in different studies. Second, different statistical and computational methods may lead to the variability. Third, both the patients infected with $\mathrm{HBV}$ and healthy individuals were included in the control group in this study.

As is known to all, analytical reproducibility is a significant challenge in MALDI protein profiling [36]. Differences in reagents and handling and changes in room temperature, pressure, and humidity may influence the (co-)crystallization step and cause day-to-day variation $[37,38]$. To improve the analytical performance, we standardized the collection and fractionation protocol and optimized parameters of MALDITOF MS. In this study, the reproducibility tests showed that the CVs of Autoflex were similar to those in other reports $[26,39]$.

\section{Conclusions}

In conclusion, we describe two classifiers, LC-NB and LCMLP, based on the serum peptide pattern for prediction of HBV-infected LC from MALDI-TOF MS in this study. The higher accuracy of the LC-NB and LC-MLP suggests that they have a great potential to emerge as noninvasive approaches in the screening of LC. Consideration has been made for further verification of their accuracy, sensitivity, and specificity in larger population from different regions and different age ranges.
Abbreviations
HBV: Hepatitis B virus
CHB: Chronic hepatitis B
SVM: Support vector machine
HCC: Hepatocellular carcinoma
ARFI: Acoustic radiation force impulse
CV: Coefficient of variance
WEKA: Waikato Environment for Knowledge Analysis
TP: $\quad$ True positive
TN: True negative
FP: $\quad$ False positive
FN: $\quad$ False negative

\author{
SE: Sensitivity \\ SP: $\quad$ Specificity \\ ACC: Accuracy \\ ROC: Receiver operator curve \\ ANN: Artificial neural network.
}

\section{Conflict of Interests}

The authors state that there is no conflict of interests regarding the publication of this paper.

\section{Authors' Contribution}

Y. Cao, K. He, and M. Cheng contributed equally to this work.

\section{Acknowledgments}

This study was supported by Grants from National Natural Science Foundation of China (nos. 81025010 and 21007092), Shandong Provincial Natural Science Foundation, China (ZR2010HQ027), National Key Technology R\&D Program (2009BAK61B04), Innovation of Methodology (2010IM030300), and China National Basic Research Program (2010CB529404).

\section{References}

[1] J. W. Kung, I. S. Currie, S. J. Forbes, and J. A. Ross, "Liver development, regeneration, and carcinogenesis," Journal of Biomedicine and Biotechnology, vol. 2010, Article ID 984248, 8 pages, 2010.

[2] A. Jemal, F. Bray, M. M. Center, J. Ferlay, E. Ward, and D. Forman, "Global cancer statistics," CA: A Cancer Journal for Clinicians, vol. 61, no. 2, pp. 69-90, 2011.

[3] A. Forner, J. M. Llovet, and J. Bruix, "Hepatocellular carcinoma," The Lancet, vol. 379, no. 9822, pp. 1245-1255, 2012.

[4] S. H. Chen, Y. F. Li, H. C. Lai et al., "Noninvasive assessment of liver fibrosis via spleen stiffness measurement using acoustic radiation force impulse sonoelastography in patients with chronic hepatitis B or C," Journal of Viral Hepatitis, vol. 19, no. 9, pp. 654-663, 2012.

[5] H. Zhang, L. Y. Wu, S. Zhang et al., "Anti-hepatitis B virus $\mathrm{X}$ protein in sera is one of the markers of development of liver cirrhosis and liver cancer mediated by HBV," Journal of Biomedicine and Biotechnology, vol. 2009, Article ID 289068, 6 pages, 2009.

[6] F. Ren, Y. Chen, Y. Wang et al., "Comparative serum proteomic analysis of patients with acute-on-chronic liver failure: alpha1-acid glycoprotein maybe a candidate marker for prognosis of hepatitis B virus infection," Journal of Viral Hepatitis, vol. 17, no. 11, pp. 816-824, 2010.

[7] J. Liu and D. Fan, "Hepatitis B in China," The Lancet, vol. 369, no. 9573, pp. 1582-1583, 2007.

[8] L. Castera, "Noninvasive methods to assess liver disease in patients with hepatitis B or C", Gastroenterology, vol. 142, no. 6, pp. 1293.e4-1302.e4, 2012.

[9] T. C. W. Poon, A. Y. Hui, H. L. Y. Chan et al., "Prediction of liver fibrosis and cirrhosis in chronic hepatitis B infection by serum proteomic fingerprinting: a pilot study," Clinical Chemistry, vol. 51, no. 2, pp. 328-335, 2005. 
[10] M. Pinzani, M. Rosselli, and M. Zuckermann, "Liver cirrhosis," Best Practice and Research, vol. 25, no. 2, pp. 281-290, 2011.

[11] K. Nightingale, M. S. Soo, R. Nightingale, and G. Trahey, "Acoustic radiation force impulse imaging: in vivo demonstration of clinical feasibility," Ultrasound in Medicine and Biology, vol. 28, no. 2, pp. 227-235, 2002.

[12] M. Fraquelli, C. Rigamonti, G. Casazza et al., "Reproducibility of transient elastography in the evaluation of liver fibrosis in patients with chronic liver disease," Gut, vol. 56, no. 7, pp. 968973, 2007.

[13] C. Henkel, K. Schwamborn, H. W. Zimmermann et al., "From proteomic multimarker profiling to interesting proteins: thymosin-beta(4) and kininogen-1 as new potential biomarkers for inflammatory hepatic lesions," Journal of Cellular and Molecular Medicine, vol. 15, no. 10, pp. 2176-2188, 2011.

[14] T. Huang, J. Wang, Y. D. Cai, H. Yu, and K. C. Chou, "Hepatitis $\mathrm{C}$ virus network based classification of hepatocellular cirrhosis and carcinoma," PLoS ONE, vol. 7, no. 4, Article ID e34460, 2012.

[15] A. M. Bozdayi, S. C. Karatayli, S. G. Alagoz et al., "Potential proteomic biomarkers in assessing liver fibrosis using SELDITOF MS," The Turkish Journal of Gastroenterology, vol. 23, no. 1, pp. 46-53, 2012.

[16] T. Liu, R. Xue, X. Huang et al., "Proteomic profiling of hepatitis $B$ virus-related hepatocellular carcinoma with magnetic beadbased matrix-assisted laser desorption/ionization time-of-flight mass spectrometry," Acta Biochimica et Biophysica Sinica, vol. 43, no. 7, pp. 542-550, 2011.

[17] B. Gangadharan, R. Antrobus, D. Chittenden et al., "New approaches for biomarker discovery: the search for liver fibrosis markers in hepatitis C patients," Journal of Proteome Research, vol. 10, no. 5, pp. 2643-2650, 2011.

[18] L. Castera and M. Pinzani, "Non-invasive assessment of liver fibrosis: are we ready?" The Lancet, vol. 375, no. 9724, pp. 1419$1420,2010$.

[19] G. Sebastiani, A. Vario, M. Guido, and A. Alberti, "Sequential algorithms combining non-invasive markers and biopsy for the assessment of liver fibrosis in chronic hepatitis B," World Journal of Gastroenterology, vol. 13, no. 4, pp. 525-531, 2007.

[20] L. Castera and M. Pinzani, "Biopsy and non-invasive methods for the diagnosis of liver fibrosis: does it take two to tango?" Gut, vol. 59, no. 7, pp. 861-866, 2010.

[21] G. M. Fiedler, A. B. Leichtle, J. Kase et al., "Serum peptidome profiling revealed platelet factor 4 as a potential discriminating peptide associated with pancreatic cancer," Clinical Cancer Research, vol. 15, no. 11, pp. 3812-3819, 2009.

[22] X. D. Zhu, W. H. Zhang, C. L. Li, Y. Xu, W. J. Liang, and P. Tien, "New serum biomarkers for detection of HBV-induced liver cirrhosis using SELDI protein chip technology," World Journal of Gastroenterology, vol. 10, no. 16, pp. 2327-2329, 2004.

[23] J. Cui, X. Kang, Z. Dai et al., "Prediction of chronic hepatitis $\mathrm{B}$, liver cirrhosis and hepatocellular carcinoma by SELDI-based serum decision tree classification," Journal of Cancer Research and Clinical Oncology, vol. 133, no. 11, pp. 825-834, 2007.

[24] C. Liu, C. Pan, J. Shen, H. Wang, and L. Yong, "MALDITOF MS combined with magnetic beads for detecting serum protein biomarkers and establishment of boosting decision tree model for diagnosis of colorectal cancer," International Journal of Medical Sciences, vol. 8, no. 1, pp. 39-47, 2011.

[25] W. Na, D. Fang-Ting, Z. Xue-Min et al., "Hydrothermal synthesis of metal-chelated magnetic nanobeads for exclusive use of peptides extraction by mass spectrum," Acta Chimica Sinica, vol. 65, no. 4, pp. 344-348, 2007.
[26] T. Liang, N. Wang, W. Li et al., "Identification of complement C3f-desArg and its derivative for acute leukemia diagnosis and minimal residual disease assessment," Proteomics, vol. 10, no. 1, pp. 90-98, 2010.

[27] M. Hall, E. Frank, G. Holmes, B. Pfahringer, P. Reutemann, and I. H. Witten, "The WEKA data mining software: an update," SIGKDD Explorations, vol. 11, no. 1, p. 10, 2009.

[28] Y. Cao, N. Wang, X. Ying et al., "BioSunMS: a plug-in-based software for the management of patients information and the analysis of peptide profiles from mass spectrometry," $B M C$ Medical Informatics and Decision Making, vol. 9, no. 1, article 13, 2009.

[29] I. A. Vergara, T. Norambuena, E. Ferrada, A. W. Slater, and F. Melo, "StAR: a simple tool for the statistical comparison of ROC curves," BMC Bioinformatics, vol. 9, article 265, 2008.

[30] V. Vapnik, Statistical Learning Theory, John Wiley \& Sons, New York, NY, USA, 1998.

[31] D. Schuppan and N. H. Afdhal, "Liver cirrhosis," The Lancet, vol. 371, no. 9615, pp. 838-851, 2008.

[32] European Association for the Study of the Liver, "EASL Clinical Practice Guidelines: management of chronic hepatitis B," Journal of Hepatology, vol. 50, no. 2, pp. 227-242, 2009.

[33] A. Tiss, C. Smith, U. Menon, I. Jacobs, J. F. Timms, and R. Cramer, "A well-characterised peak identification list of MALDI MS profile peaks for human blood serum," Proteomics, vol. 10, no. 18, pp. 3388-3392, 2010.

[34] M. Dakna, K. Harris, A. Kalousis et al., "Addressing the challenge of defining valid proteomic biomarkers and classifiers," BMC Bioinformatics, vol. 11, article 594, 2010.

[35] L. Wuju and X. Momiao, "Tclass: tumor classification system based on gene expression profile," Bioinformatics, vol. 18, no. 2, pp. 325-326, 2002.

[36] J. Albrethsen, "Reproducibility in protein profiling by MALDITOF mass spectrometry," Clinical Chemistry, vol. 53, no. 5, pp. 852-858, 2007.

[37] S. Garbis, G. Lubec, and M. Fountoulakis, "Limitations of current proteomics technologies," Journal of Chromatography A, vol. 1077, no. 1, pp. 1-18, 2005.

[38] C. D. Calvano, A. Aresta, M. Iacovone et al., "Optimization of analytical and pre-analytical conditions for MALDI-TOF-MS human urine protein profiles," Journal of Pharmaceutical and Biomedical Analysis, vol. 51, no. 4, pp. 907-914, 2010.

[39] E. Orvisky, S. K. Drake, B. M. Martin et al., "Enrichment of low molecular weight fraction of serum for MS analysis of peptides associated with hepatocellular carcinoma," Proteomics, vol. 6, no. 9, pp. 2895-2902, 2006. 

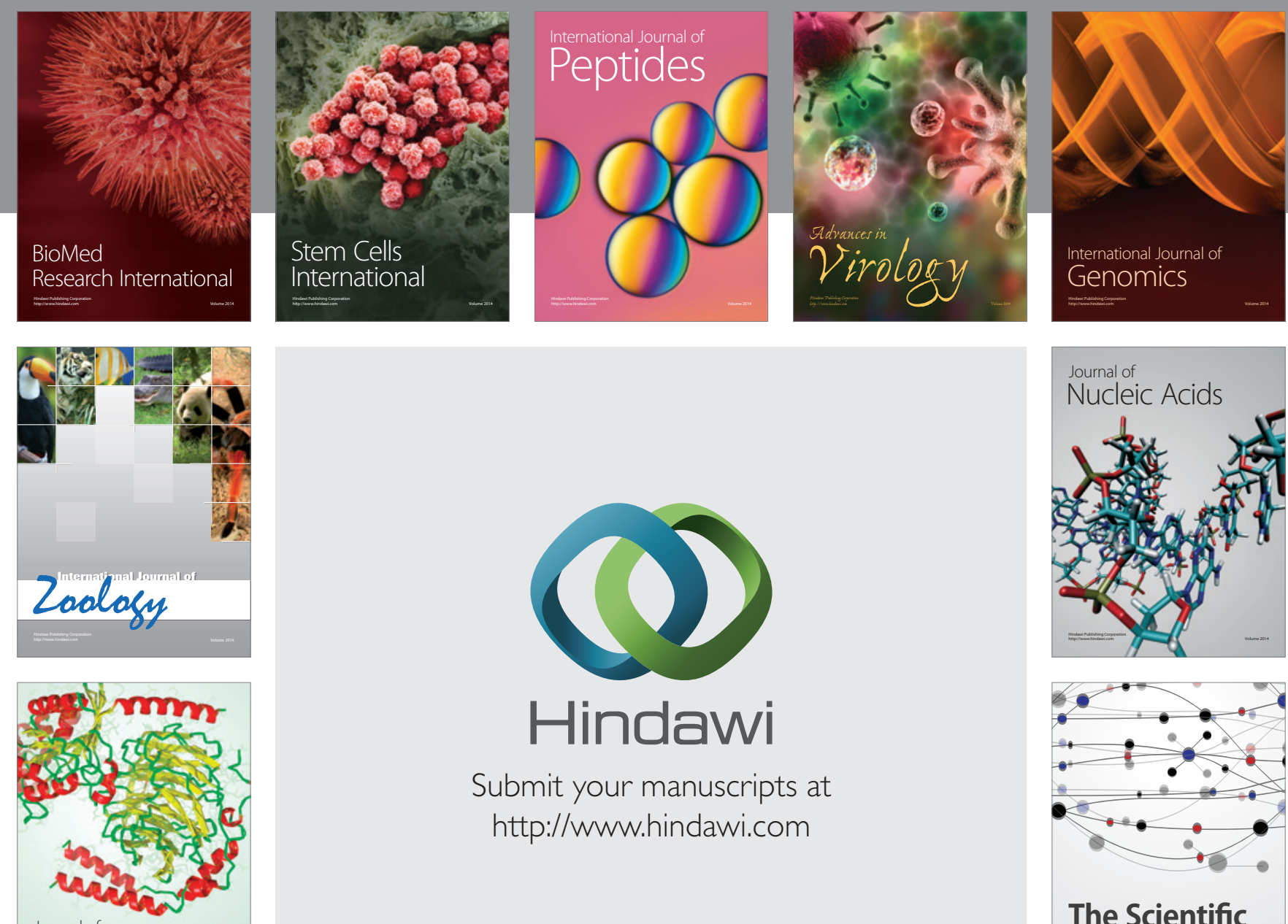

Submit your manuscripts at

http://www.hindawi.com

Journal of
Signal Transduction
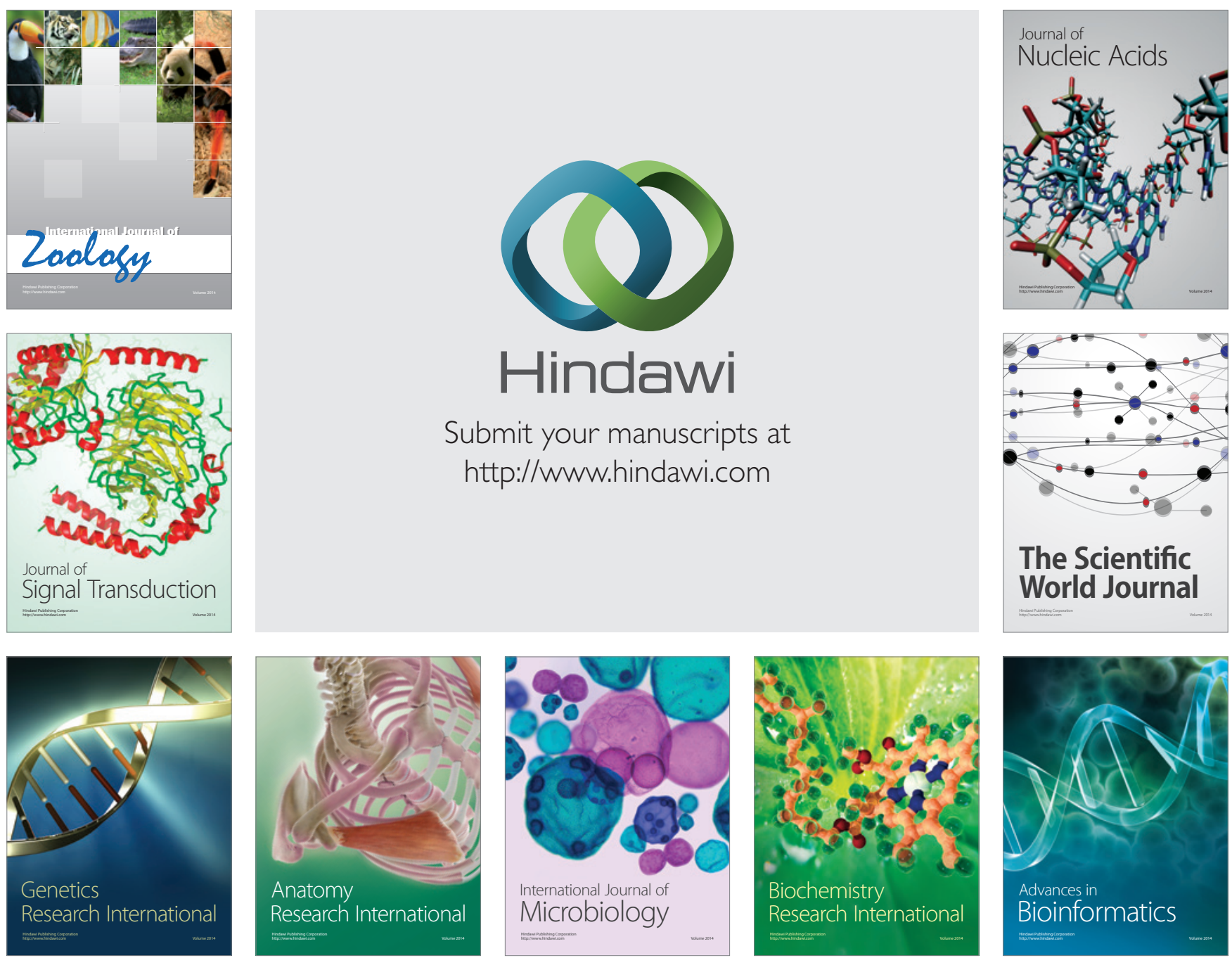

The Scientific World Journal
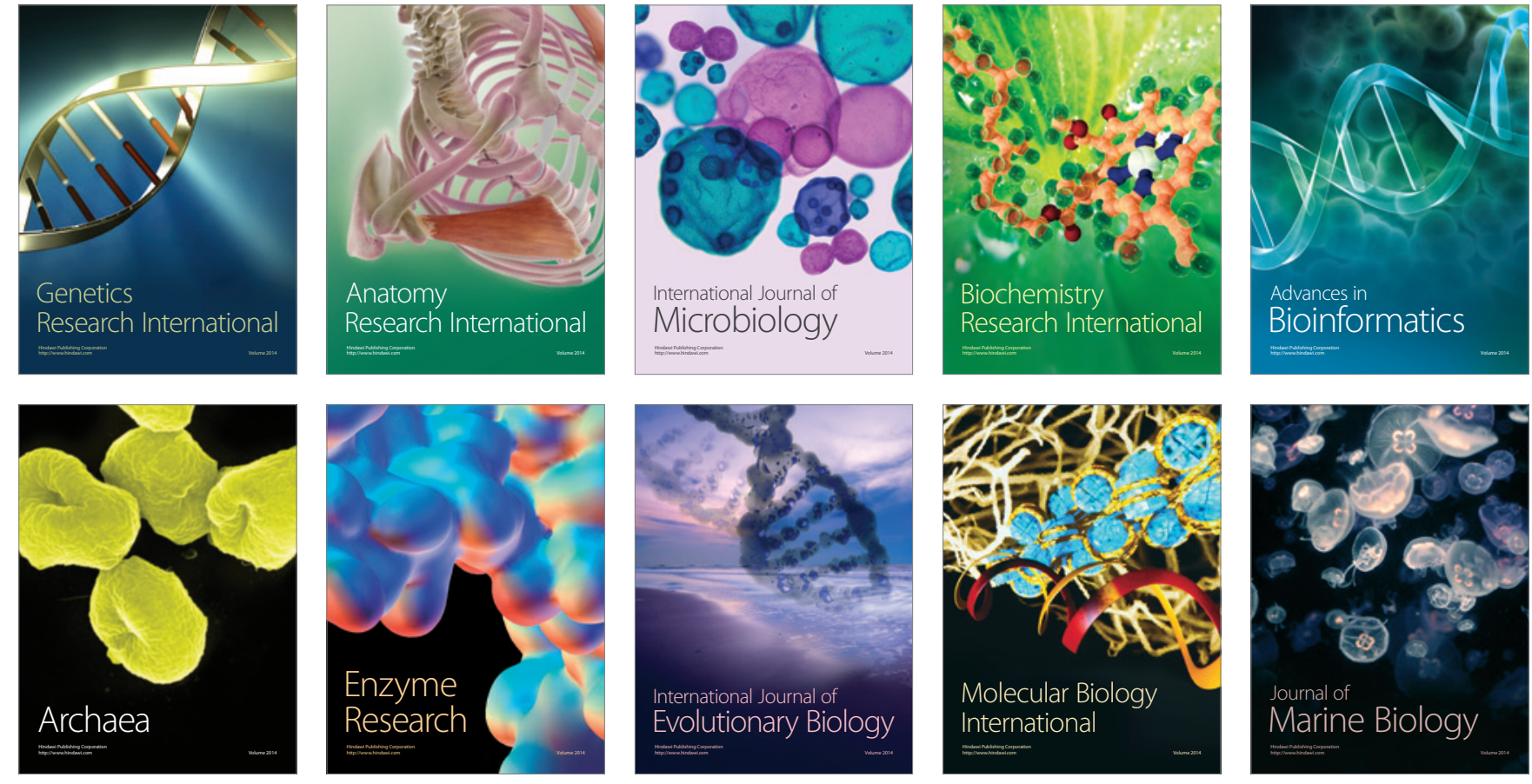